\title{
Malakoplakia Affecting the Umbilical Cord
}

\author{
Song-Hee Han · Mee Joo · Sunhee Chang · Han-Seong Kim \\ Department of Pathology, Inje University Ilsan Paik Hospital, Inje University College of Medicine, Goyang, Korea
}

Malakoplakia, meaning soft (malako) plaque (plakia), is a rare benign inflammatory lesion. The disease was initially described in the bladder by Michaelis and Gutmann ${ }^{1}$ in 1902 and named by von Hansemann ${ }^{2}$ in 1903 . Since its initial description, many cases have been described in numerous anatomic locations including the genitourinary tract, gastrointestinal tract, pancreas, liver, oropharynx, retroperitoneum, thyroid gland, lymph nodes, lung, bone/joint, brain, skin, and other tissues. ${ }^{3-5}$ To our knowledge, this is the first case of malacoplakia affecting the umbilical cord.

\section{CASE REPORT}

A 33-year-old primigravida and nullipara woman in preterm labor at 32 weeks of gestation was referred to our hospital. Upon presentation, she had a temperature of $37.3^{\circ} \mathrm{C}$, a pulse rate of 108 beats per minute, and leukocytosis (white blood cells count $>22,000 \mathrm{~mm}^{2}$ ). Vaginal examination revealed no pooling of fluid. She gave birth to a 2,000 $\mathrm{g}$ female infant by vaginal delivery, with Apgar scores of 7 and 9 at 1 and 5 minutes, respectively.

Grossly, the placenta was $18 \times 14 \times 2.5 \mathrm{~cm}$ and $510 \mathrm{~g}$. The attached cord measured $30 \mathrm{~cm}$ in length and $2 \mathrm{~cm}$ and $1.3 \mathrm{~cm}$ in maximal and minimal diameters, respectively. The middle portion of the umbilical cord was focally enlarged, measuring $2 \mathrm{~cm}$ in length and $2 \mathrm{~cm}$ in diameter. The cut surface showed an ill-defined white to brown horseshoe-shaped area surrounding the umbilical vessels, measuring $2 \mathrm{~cm}$ in length and $0.2 \mathrm{~cm}$

\section{Corresponding Author}

Hanseong Kim, M.D.

Department of Pathology, Inje University Ilsan Paik Hospital, Inje University College of Medicine, 170 Juhwa-ro, Ilsanseo-gu, Goyang 411-706, Korea

Tel: +82-31-910-7142, Fax: +82-31-910-7139, E-mail: hskim@paik.ac.kr

Received: December 11, 2014 Revised: February 4, 2015

Accepted: February 4, 2015 in thickness (Fig. 1A). The chorionic plate had a patchy appearance and was covered by a purulent exudate.

Microscopically, there was a horseshoe-shaped calcified area located within the Wharton's jelly. The calcified area consisted of multiple, small, round to oval, and calcified bodies (calcospherites), which were both intra- and extracellular. Many of the inclusions were confirmed to be within macrophages on CD68 immunohistochemical stain. The majority of the lesion was necrotic and contained an inflammatory infiltrate composed mainly of neutrophils and macrophages. The macrophages had abundant eosinophilic cytoplasm with eccentric, hyperchromatic, and round nuclei (Fig. 1B). Concentrically laminated, roundovoid, and basophilic calcified bodies, which are referred to as Michaelis-Gutmann bodies, were also seen (Fig. 1C, D). These bodies stained positive for von-Kossa and periodic acid-Schiff. Acute inflammation of the choriodecidua, amnion, and chorionic plate was noted. Based on the above findings, the diagnosis of malakoplakia of the umbilical cord, acute chorioamnionitis, and funisitis was made. Unfortunately, intracellular and extracellular bacterial organisms were not revealed on special stains.

\section{DISCUSSION}

The pathogenesis of malakoplakia is thought to be related to infections and/or immunosuppressed states, such as human immunodeficiency virus infection, cancer chemotherapy, post organ or bone marrow transplant states, and congenital immunodeficiency associated with an acquired bactericidal defect of macrophages. ${ }^{6}$ The presence of a bacterial infection is clear in almost all reported cases, regardless of the site of organ involvement. Klebsiella pneumoniae and Escherichia coli are the most common cultured microorganisms in lesions of malakoplakia, although other gram-negative, gram-positive, and acid-fast bacilli have been implicated. ${ }^{5}$ In addition, the patients of several prior re- 



Fig. 1. Representative macroscopic and microscopic images of the lesion. (A) The middle portion of umbilical cord is focally enlarged, inside which an ill-defined white to brown horseshoe-shaped area (arrowhead) surrounding the umbilical vessels is noted. (B) The concentrically laminated, round-ovoid, basophilic calcified bodies, referred to as Michaelis-Gutmann bodies, are seen within macrophages (arrowhead). (C) This lesion is composed of multiple, small, round to oval, calcified bodies (calcospherites), Michaelis-Gutmann bodies (arrowheads), and an inflammatory infiltrate containing mainly necrotic macrophages. (D) The Michaelis-Gutmann bodies (arrowheads) are also observed in the noncalcified area.

ports have been immunosuppressed or had an autoimmune disease, neoplasm, or organ transplant. Thorning and Vracko ${ }^{6}$ suggested that malakoplakia may be caused by impaired phagocytosis and a specific defect in the acidification of lysosomal vacuoles containing partially digested bacterial particulates, and therefore, as mentioned above, macrophages are able to ingest but not kill specific organisms.

While pregnancy is not an immunosuppressed state, immunologic changes during pregnancy may increase the susceptibility to certain pathogens, including viruses, bacteria, and parasites. Therefore, pregnancy is associated with an increased risk of infection. Given that our patient presented with a mild fever, leukocytosis, and tachycardia, it was highly suspected to be an infection. Clinical and histological chorioamnionitis was confirmed. The pathogenesis of chorioamnionitis is marked by the passage of infectious organisms to the chorioamnion and/or the umbilical cord of the placenta. ${ }^{7}$ This passage occurs most commonly by an ascending infection from the lower genital tract. ${ }^{7}$ In pregnancy, two cases of malakoplakia have been reported. One case involved the pelvic peritoneum and the other involved the cerebrum. ${ }^{8,9}$ A urinary tract infection was the cause of infection in both cases. ${ }^{8,9}$ Unfortunately, the offending organism in the present case was not isolated making the cause unclear.

During normal pregnancy, there is an increase in innate immune cells, such as macrophages and natural killer cells. These macrophages may be important for local immune function as well as the placental development by promoting trophoblast recruitment, spiral artery remodeling, and angiogenesis. ${ }^{10}$ However, it is currently unknown why some patients develop the dysfunctional changes of macrophages that can predispose pa- 
tients to malakoplakia.

This is the first case of malakoplakia affecting the umbilical cord. Our findings illustrate that in cases with clinical features associated with clinical chorioamnionitis including maternal fever and tachycardia (>100/min), fetal tachycardia (>160/min) and purulent or foul-smelling amniotic fluid, malakoplakia should be suspected.

\section{Conflicts of Interest}

No potential conflict of interest relevant to this article was reported.

\section{REFERENCES}

1. Michaelis L, Gutmann C. Uber einschlusse in blasentumoren. Z Klin Med 1902; 47: 208-15.

2. Von Hansemann D. Uber malakoplakie der harnblase. Virchows Arch Pathol Anat Physiol Klin Med 1903; 173: 302-8.

3. Afonso JP, Ando PN, Padilha MH, Michalany NS, Porro AM. Cutaneous malakoplakia: case report and review. An Bras Dermatol 2013;
88: 432-7.

4. Hamvas A, Detre Z, Szinnay G. Malakoplakia of kidney and urinary tract: report of a case of fatal outcome. Int Urol Nephrol 1981; 13: 55-63.

5. Wagner D, Joseph J, Huang J, Xu H. Malakoplakia of the prostate on needle core biopsy: a case report and review of the literature. Int J Surg Pathol 2007; 15: 86-9.

6. Thorning D, Vracko R. Malakoplakia: defect in digestion of phagocytized material due to impaired vacuolar acidification? Arch Pathol 1975; 99: 456-60.

7. Czikk MJ, McCarthy FP, Murphy KE. Chorioamnionitis: from pathogenesis to treatment. Clin Microbiol Infect 2011; 17: 1304-11.

8. Rose G, Morrison EA, Kirkham N, Machling R. Malakoplakia of the pelvic peritoneum in pregnancy: case report. Br J Obstet Gynaecol 1985; 92: 170-2.

9. Blumbergs PC, Hallpike JF, McClure J. Cerebral malakoplakia. J Clin Pathol 1981; 34: 875-8.

10. Svensson-Arvelund J, Ernerudh J, Buse E, et al. The placenta in toxicology. Part II: systemic and local immune adaptations in pregnancy. Toxicol Pathol 2014; 42: 327-38. 